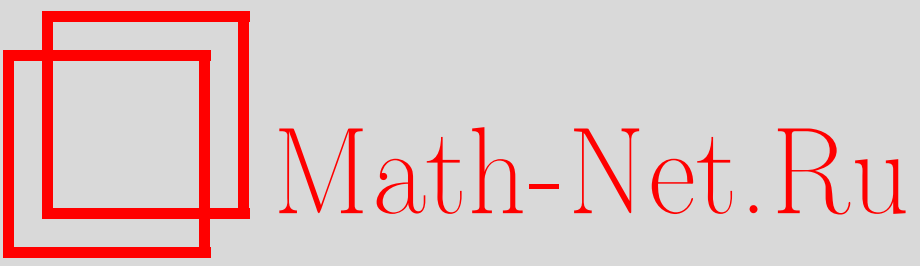

И. Е. Павлюкевич, K расчету вероятностных характеристик некоторых оптимальных моментов остановки, УМН, 1997, том 52, выпуск 1, 233-234

DOI: https://doi.org/10.4213/rm813

Использование Общероссийского математического портала Math-Net.Ru подразумевает, что вы прочитали и согласны с пользовательским соглашением

http://www . mathnet.ru/rus/agreement

Параметры загрузки:

IP : 54.162 .85 .209

26 апреля 2023 г., 03:39:53 


\section{К РАСЧЕТУ ВЕРОЯТНОСТНЫХ ХАРАКТЕРИСТИК НЕКОТОРЫХ ОПТИМАЛЬНЫХ МОМЕНТОВ ОСТАНОВКИ}

И.Е. ПАВЛЮКЕВИЧ

В работе [1] М. Жанблан-Пике и А. Н. Ширяев рассмотрели, следуя [2], модель эволюции капитала фирмы $X=\left(X_{t}\right)_{t \geqslant 0}$, предполагая, что

$$
d X_{t}=\mu d t+\sigma d W_{t}-d Z_{t}
$$

где $W=\left(W_{t}\right)_{t \geqslant 0}-$ стандартньй винеровский процесс, коэффициенты $\mu>0, \sigma>0$. Неотрицательный неубьвающий неупреждающий процесс $Z=\left(Z_{t}\right)_{t \geqslant 0}$ характеризует стратегию выплаты фирмой дивидендов.

Предполагается, что началњный капитал $X_{0}=x \geqslant 0$, в момент первого обращения в нуль процесса $X$ происходит разорение, и $d X_{t}=d Z_{t}=0$ для $t \geqslant \tau$, где $\tau$ - момент разорения, $\tau=\inf \left\{t: X_{t}=0\right\}$.

В [1] были найдены оптималшные дивидендные процессы $Z=\left(Z_{t}\right)_{t \geqslant 0}$, т.е. те, которые доставляют sup функции эффективности функционирования фирмы (см. [2])

$$
V(x)=\sup \mathrm{E}_{x} \int_{0}^{\infty} e^{-\lambda t} d Z_{t}=\sup \mathrm{E}_{x}\left[Z_{0}+\int_{(0, \infty)} e^{-\lambda t} d Z_{t}\right], \quad \lambda>0,
$$

в трех случаях $\mathbf{A}, \mathbf{B}, \mathbf{C}$ (см. далее формулировку теоремы). Следуя общей идеологии [1], в настоящей работе вычислены математические ожидания момента $\tau$ разорения фирмы в каждом из этих трех случаев.

Tеорема. Обозначим $\theta=2 \mu / \sigma^{2}$.

A. Пусть процесс $Z=\left(Z_{t}\right)_{t \geqslant 0}$ таков, ито $d Z_{t}=u\left(X_{t}\right) d t, \quad Z_{0}=Z_{0}(x)$, где $u=u(x), Z_{0}=Z_{0}(x)$ - произвольнье измеримье функиии, причем $0 \leqslant u(x) \leqslant K<\infty$, $0 \leqslant Z_{0}(x) \leqslant x$. Тогда

1) если $K \leqslant K_{*}$, то при наличии начального капитала $X_{0}=x$ дивидендь выплачиваются сразу (см. [1]) и, следовательно, $m_{\mathbf{A}}(x)=0$ для всех $x \geqslant 0$;

2) если $K>K_{*}$, то

$$
m_{\mathbf{A}}(x)= \begin{cases}-\frac{x}{\mu}+\frac{1}{\theta \mu} e^{\theta \widetilde{x}}-\frac{1}{\theta \mu} e^{\theta(\widetilde{x}-x)}, & x \leqslant \widetilde{x}, \\ -\frac{\widetilde{x}}{\mu}+\frac{1}{\theta \mu} e^{\theta \widetilde{x}}-\frac{1}{\theta \mu}, & x>\widetilde{x} .\end{cases}
$$

Здесь $K_{*}-$ корень уравнения $\lambda / K_{*}=\left|\rho\left(K_{*}\right)\right|, \widetilde{x}=\widetilde{x}\left(K_{*}\right)-$ корень уравнения $\operatorname{th}(\Delta \widetilde{x})=$ $(2(\lambda+K \rho) \Delta) /(\mu(\lambda+K \rho)+2 \lambda \rho), \rho=\rho(K)=(K-\mu) / 2-\sqrt{(K-\mu)^{2} / 4+\lambda}, \Delta=\sqrt{\mu^{2} / 4+\lambda}$. В. Пусть прочесс $Z=\left(Z_{t}\right)_{t \geqslant 0}$ принадлежит классу процессов вида

$$
Z_{t}=\sum_{i \geqslant 0} e^{-\lambda T_{i}} \zeta_{i} I\left(T_{i} \leqslant t\right)
$$

где $0=T_{0}<T_{1}<T_{2}<\cdots$ - моменты выплаты дивидендов, а $\zeta_{0}, \zeta_{1}, \ldots$ - неотричательные величины выплачиваемых дивидендов. При этом предполагается, что за каждую выплату дивидендов взимается плата $\gamma>0$. Тогда

$$
m_{\mathbf{B}}(x)= \begin{cases}-\frac{x}{\mu}+\frac{1}{\mu} \frac{\widetilde{b}-\widetilde{a}}{e^{-\theta \widetilde{a}}-e^{-\theta \widetilde{b}}}\left(1-e^{-\theta x}\right), \quad x \leqslant \widetilde{b}, \\ -\frac{\widetilde{a}}{\mu}+\frac{1}{\mu} \frac{\widetilde{b}-\widetilde{a}}{e^{-\theta \widetilde{a}}-e^{-\theta \widetilde{b}}}\left(1-e^{-\theta \widetilde{b}}\right), \quad x>\widetilde{b} .\end{cases}
$$


Здесь $\widetilde{a}$ и $\widetilde{b}-$ величинь, определяюшиеся из системь (3.14) в [1].

C. Пусть $Z=\left(Z_{t}\right)_{t \geqslant 0}-$ произвольный неотрицательный неубывающий неупреждающий прощесс, непрерьвный справа при $t>0$. Тогда

$$
m_{\mathbf{C}}(x)= \begin{cases}-\frac{x}{\mu}+\frac{1}{\theta \mu} e^{\theta \bar{x}}-\frac{1}{\theta \mu} e^{\theta(\bar{x}-x)}, & x \leqslant \bar{x}, \\ -\frac{\bar{x}}{\mu}+\frac{1}{\theta \mu} e^{\theta \bar{x}}-\frac{1}{\theta \mu}, & x>\bar{x} .\end{cases}
$$

Здесь $\bar{x}-$ корень уравнения $\operatorname{th}(\Delta \bar{x})=\mu \Delta /\left(2 \Delta^{2}-\lambda\right)$.

Функции $m_{\mathbf{A}}, m_{\mathbf{B}}, m_{\mathbf{C}}$ были найдены как решения следующих краевых задач. Пусть $\mathscr{L}=\mu \frac{d}{d x}+\frac{\sigma^{2}}{2} \frac{d^{2}}{d x^{2}}$, тогда

$$
\begin{gathered}
(\mathbf{A}) \quad\left\{\begin{array}{l}
\mathscr{L} m_{\mathbf{A}}(x)=-1, x \in(0, \widetilde{x}), \\
m_{\mathbf{A}}(0)=0, \\
(\mu-K) m_{\mathbf{A}}^{\prime}(\widetilde{x})+\frac{\sigma^{2}}{2} m_{\mathbf{A}}^{\prime \prime}(\widetilde{x})=-1,
\end{array}\right. \\
\text { (B) }\left\{\begin{array} { l } 
{ \mathscr { L } m _ { \mathbf { B } } ( x ) = - 1 , \quad x \in ( 0 , \widetilde { b } ) , } \\
{ m _ { \mathbf { B } } ( 0 ) = 0 , } \\
{ m _ { \mathbf { B } } ( \widetilde { b } - ) = m _ { \mathbf { B } } ( \widetilde { a } ) , }
\end{array} \quad ( \mathbf { C } ) \quad \left\{\begin{array}{l}
\mathscr{L} m_{\mathbf{C}}(x)=-1, x \in(0, \bar{x}), \\
m_{\mathbf{C}}(0)=0, \\
m_{\mathbf{C}}^{\prime}(\bar{x})=0 .
\end{array}\right.\right.
\end{gathered}
$$

Краевая задача $(\mathbf{C})$ может быть получена из задач $(\mathbf{A})$ и $(\mathbf{B})$, соответственно, предельным переходом при $K \rightarrow \infty$ и $\gamma \rightarrow 0$. При этом $\widetilde{x}=\widetilde{x}(K) \rightarrow \bar{x}, \widetilde{a}, \widetilde{b} \rightarrow \bar{x}$ и $m_{\mathbf{A}}, m_{\mathbf{B}} \rightarrow m_{\mathbf{C}}$. Остается лишь добавить, что во всех случаях математическое ожидание времени разорения фирмы конечно.

\section{СПИСОК ЛИТЕРАТУРЫ}

[1] ЖКанблан-Пике М., Ширяев А. Н. // УМН. 1995. Т. 50. № 2. С. 25-46. [2] Radner R., Shepp L. Risk vs. Profit-Potential; A Model for Corporate Strategy // Preprint. 